\title{
Synthesis of hexagonal close-packed gold nanostructures
}

\author{
Xiao Huang', Shaozhou Li', Yizhong Huang', Shixin Wu', Xiaozhu Zhou', Shuzhou Li', Chee Lip Gan', \\ Freddy Boey ${ }^{1,2}$, Chad A. Mirkin³ \& Hua Zhang ${ }^{1,2}$
}

Solid gold is usually most stable as a face-centred cubic $(f c c)$ structure. To date, no one has synthesized a colloidal form of Au that is exclusively hexagonal close-packed ( $h c p$ ) and stable under ambient conditions. Here we report the first in situ synthesis of dispersible hcp Au square sheets on graphene oxide sheets, which exhibit an edge length of 200-500 nm and a thickness of $\sim 2.4 \mathrm{~nm}$ ( $16 \mathrm{Au}$ atomic layers). Interestingly, the Au square sheet transforms from hcp to a fcc structure on exposure to an electron beam during transmission electron microscopy analysis. In addition, as the square sheet grows thicker (from $\sim 2.4$ to $6 \mathrm{~nm}$ ), fcc segments begin to appear. A detailed experimental analysis of these structures shows that for structures with ultrasmall dimensions (for example, $<\sim 6 \mathrm{~nm}$ thickness for the square sheets), the previously unobserved pure hcp structure becomes stable and isolable.

\footnotetext{
'School of Materials Science and Engineering, Nanyang Technological University, 50 Nanyang Avenue, Singapore 639798, Singapore. ${ }^{2}$ Center for Biomimetic Sensor Science, Nanyang Technological University, 50 Nanyang Drive, Singapore 637553, Singapore. ${ }^{3}$ Department of Chemistry and International Institute for Nanotechnology, Northwestern University, 2145 Sheridan Road, Evanston, Illinois 60208-3113, USA. Correspondence and requests for materials should be addressed to H.Z. (email: hzhang@ntu.edu.sg) or to C.A.M. (email: chadnano@northwestern.edu).
} 
A nisotropic noble metal nanoparticles (gold $(\mathrm{Au})$ and silver $(\mathrm{Ag}))$ are a class of materials with chemical and physical properties that directly correlate with their structures. They have been used for many applications in biosensing, intracellular gene regulation, photonics and catalysis ${ }^{1-5}$. Colloids of well-dispersed spherical $\mathrm{Au}$ nanoparticles were successfully synthesized decades ago by refluxing $\mathrm{HAuCl}_{4}$ with sodium citrate ${ }^{6}$. These procedures have been refined, and now can reliably be used to prepare monodisperse samples of particles in the 2- to 200-nm diameter range. In addition to particle size control, particle shape control is also important. Over the past 10 years, significant strides have been made in synthesizing anisotropic Au nanostructures (AuNSs) ${ }^{7-9}$. To realize anisotropic growth, template-mediated syntheses are some of the most effective approaches. Examples include the surfactantdirected soft-templating method $^{7}$ and the biomolecule-facilitated hard-templating approach ${ }^{10}$. More recently, ultrathin single-crystalline $\mathrm{Au}$ nanowires (AuNWs) were synthesized by slow reduction of $\mathrm{HAuCl}_{4}$ in the presence of 1-amino-9-octadecene, which serves as a soft template for the growth of the wires ${ }^{5,11,12}$.

Graphene, a single atomic layer of graphite, has attracted tremendous attention because of its exceptional physical properties and promise in various applications ${ }^{13,14}$. One of its derivatives, graphene oxide (GO), is an extensively oxidized two-dimensional (2D) carbon sheet, which is suitable for chemical modification and subsequent device incorporation ${ }^{15}$. These structures also have interesting properties. To date, researchers have used them to synthesize nanoparticles, buttheresulting structures have been mostlyspherical ${ }^{16}$ or lack good crystallinity ${ }^{17}$.

Herein, we report the in situ synthesis of pure hexagonal closepacked ( $h c p$ ) Au square sheets (AuSSs) on GO. The AuSSs, with an edge length of $200-500 \mathrm{~nm}$ and a thickness of $\sim 2.4 \mathrm{~nm}(\sim 16$ $\mathrm{Au}$ atomic layers), consist of a unique atomic arrangement of $\mathrm{Au}$. A detailed experimental analysis of this structure shows that for a structure with ultrasmall dimensions (for example, less than $\sim 6-\mathrm{nm}$ thickness for the AuSSs), the pure $h c p$ phase becomes stable and isolable at ambient conditions. In addition, we show that the crystal lattice of the AuSSs transforms from the hcp phase to a face-centred cubic $(f c c)$ structure on exposure to a transmission electron microscope (TEM) beam.

\section{Results}

Synthesis and characterization of AuSSs. The AuSSs were synthesized by heating a solution containing GO sheets, 4-mM $\mathrm{HAuCl}_{4}$ and 140-mM 1-amino-9-octadecene, $\mathrm{CH}_{3}\left(\mathrm{CH}_{2}\right)_{7} \mathrm{CH}=\mathrm{C}$ $\mathrm{H}\left(\mathrm{CH}_{2}\right)_{8} \mathrm{NH}_{2}$, in a solution of hexane and ethanol $(\mathrm{v} / \mathrm{v}=23: 2)$ at $55^{\circ} \mathrm{C}$ for $16 \mathrm{~h}$ (Supplementary Fig. S1). Interestingly, the obtained pseudo-square-shaped nanostructures exhibit an edge length between 200 and $500 \mathrm{~nm}$, as determined by TEM analysis (Fig. 1a). The Au composition of the as-prepared nanostructures was confirmed by energy dispersive X-ray analysis (Supplementary Fig. S2). The thickness of a typical AuSS, which is estimated by measuring the width of folded edges of an AuSS (Supplementary Fig. S3), is $2.4 \pm 0.7 \mathrm{~nm}$. Note that a similar method has been used to characterize monolayer and bilayer sheets of graphene ${ }^{13}$. Atomic force microscopy measurements for a typical AuSS on GO (Supplementary Fig. S4) indicate a thickness of $4 \mathrm{~nm}$, which is $\sim 1.6 \mathrm{~nm}$ greater than the thickness of the AuSS core, as determined by TEM. This apparent increase in thickness is because the alkylamine molecules, adsorbed on the AuSS surface ${ }^{18}$, are invisible by TEM but are clearly present based on X-ray photoelectron spectroscopy (Supplementary Fig. S5). Surprisingly, these AuSSs are hcp (2H type) nanostructures, as evidenced by selected area electron diffraction (SAED) data (Fig. 1c,d). For example, a typical AuSS oriented on GO, with its square basal plane normal to the electron beam (e-beam), exhibits two rings assigned to the GO $\{100\}$ and $\{110\}$ planes with lattice spacings of 2.2 and $1.3 \AA$, respectively (Fig. 1c; note that directly dropping the sample solution onto a copper grid often results in multilayer GO deposition). The remaining diffraction spots for the AuSS fit the hcp Au lattice structure (2H type, with a symmetry of $\mathrm{P} 63 / \mathrm{mmc})$, viewed along the $[110]_{h}$ direction. The SAED pattern taken at $[320]_{h}$ zone axis (Fig. 1d) was also obtained by tilting an AuSS along the $(1 \overline{1} 1)_{h}$ reflection by $\sim 19^{\circ}$ with respect to the $[110]_{h}$ zone axis, in agreement with the theoretical angle between $[320]_{h}$ and $[110]_{h}$, that is, $19^{\circ}$. For further comparison and confirmation, SAED patterns along the $[110]_{h}$ and $[320]_{h}$ zone axes were simulated (Supplementary Fig. S6), which are consistent with the patterns obtained experimentally (Fig. 1c,d). X-ray diffraction (XRD) data for AuSSs on GO, deposited on a glass slide, are also consistent with the $h c p$ assignment (Supplementary Fig. S7). The peaks at $\sim 37$ and $\sim 40^{\circ}$ are associated with the $\mathrm{Au}(002)_{h}$ and $(101)_{h}$ planes, respectively, for a $2 \mathrm{H}-h c p$ phase (the simulated unit cell parameters are $a=2.96 \AA$ and $c=4.84 \AA$ ). The remaining peaks are from $f c c \mathrm{Au}$, because of the presence of spherical Au nanoparticles (byproducts of the reaction forming the AuSSs, see the several dark Au dots with size of $10-25 \mathrm{~nm}$ in Fig. 1a). Note that the peaks for $h c p$ phase appear very weak as compared with the $f c c$ phase. This observation, in part, is because of their anisotropic properties. The AuSSs on GO sheets deposited on a substrate for XRD were so highly oriented that not all of the crystal planes showed strong reflections; however, the pseudo-spherical $f c c$ Au nanoparticles were randomly distributed on the substrate, giving rise to the strong reflections from different crystal planes (Supplementary Fig. S7a). In addition, the reflections from the $(110)_{h}$ square basal planes of AuSSs are barely observable because of the ultrathin nature of the AuSSs. The 2.4-nm dimension, normal to the (110) plane, results in a weak and broad reflection peak based on Sherrer's theory ${ }^{19}$, whereas the $f c c$ Au nanoparticles, which are 10-25 nm in diameter, produce strong reflections. For comparison, a concentrated solution (similar to a paste) of AuSSs was deposited on a glass slide, so that the AuSSs did not show the preferred orientation. As a result, stronger signals for $h c p$ phase, for example, those for the $(002)_{h}$ and $(101)_{h}$ planes, were observed with considerable broadening and overlapping (Supplementary Fig. S7b). The high-resolution TEM (HRTEM) image of a small section of a typical AuSS matches the lattice pattern of a $2 \mathrm{H}-h c p$ Au along the $[110]_{h}$ zone axis (Fig. 1b). A series of simulated HRTEM images of $h c p$ Au sheets, which vary in thickness, are shown in Supplementary Figure S8. The calculated lattice spacings for $(002)_{h}$ and $(1 \overline{1} 1)_{h}$ based on the XRD results are 2.42 and $2.27 \AA$, respectively, which are in agreement with the measured values in the HRTEM image of the AuSS, Figure 1b. The calculated lattice spacing for the $(110)_{h}$ basal planes is $1.48 \AA$; thus, an AuSS with a thickness of $2.4 \mathrm{~nm}$ contains $\sim 16 \mathrm{Au}$ atomic layers. The AuSSs are not perfect squares. The two opposite angles along the $[002]_{h}$ diagonal $\left(93^{\circ}\right)$ are slightly larger than those along the $[1 \overline{1} 0]_{h}$ diagonal $\left(87^{\circ}\right)$, which matches well with the interplane angles of $(1 \overline{1} 2)_{h} /(\overline{1} 12)_{h}\left(93.3^{\circ}\right)$ and $(\overline{1} 1 \overline{2})_{h} /(\overline{1} 12)_{h}\left(86.7^{\circ}\right)$, respectively. Therefore, crystal models, with $2 \mathrm{D}$ and $3 \mathrm{D}$ views, for the AuSSs are proposed in the inset of Figure 1a, indicating that the square basal plane in each nanostructure is normal to the $[110]_{h}$ direction, with $\mathrm{ABAB}$ stacking along the $[001]_{h}$ direction, and four sides enclosed by $\{\overline{1} 12\}_{h}$ planes.

When the aforementioned growth solution for the AuSSs on $\mathrm{GO}$ was heated at $55^{\circ} \mathrm{C}$ for $28 \mathrm{~h}$, the AuSSs became thicker $(6.0 \pm 0.7 \mathrm{~nm}$, Supplementary Fig. S9b), but exhibit a similar square-like shape with truncated corners (Supplementary Fig. S9). Alternating dark and bright strips are observed along one of the diagonals (Supplementary Fig. S9a), indicating the presence of stacking faults or twin defects. Indeed, HRTEM images and XRD pattern confirm the mixed stacking of $h c p$ and $f c c$ planes (Supplementary Figs. S7c and S9c), suggesting that as the AuSS grows thicker (from about 2.4 to $6.0 \mathrm{~nm}$ ) the pure $h c p$ phase becomes less stable. 
a

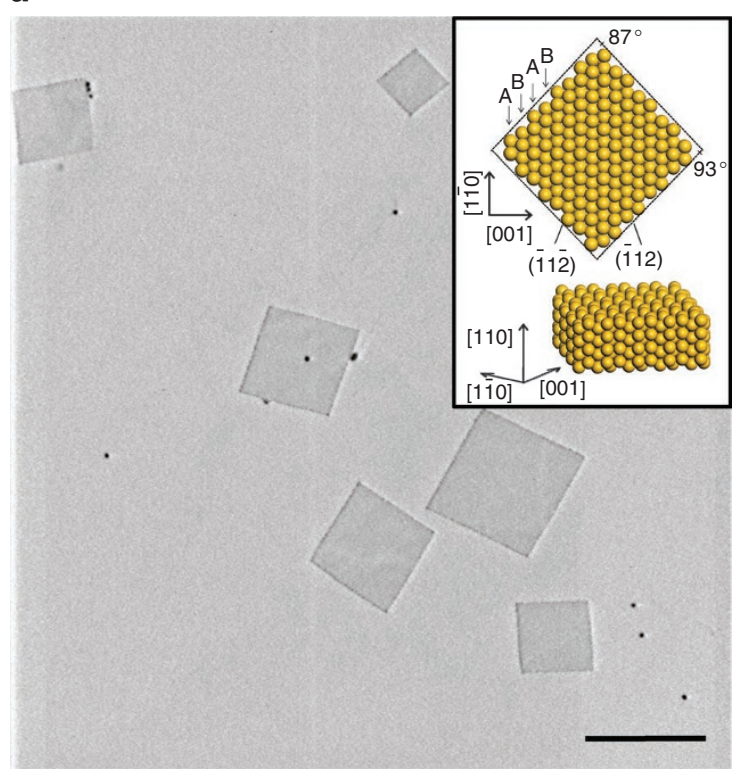

b
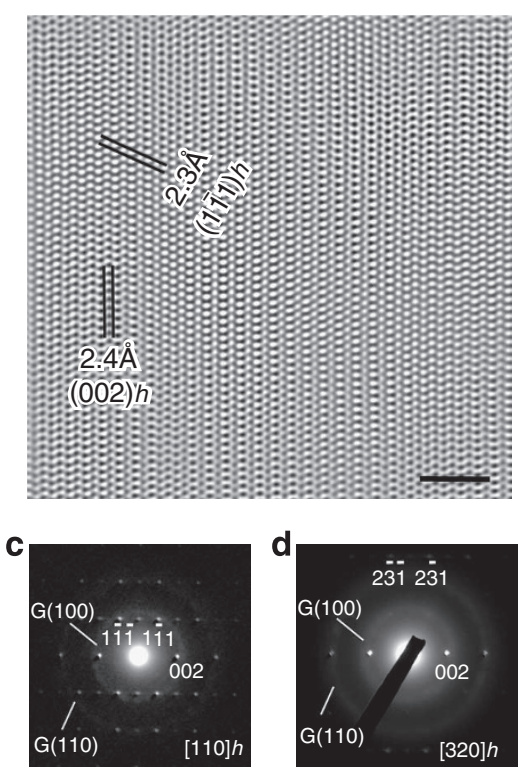

Figure 1 | TEM analysis of Au square sheets. (a) TEM image of 2.4-nm thick AuSSs on a GO surface (scale bar, $500 \mathrm{~nm}$ ). The black dots are nanoparticle byproducts, that is, spherical Au nanoparticles (approximately 10-25 nm). Inset: crystallographic models for a typical AuSS with its basal plane along the $[110]_{h}$ zone axis, showing ABAB stacking along the $[001]_{h}$ direction. (b) HRTEM image of a small region of a typical AuSS oriented normal to $[110]_{h}$, as indicated from the SAED in c (scale bar, $2 \mathrm{~nm}$ ). (c) SAED pattern of an AuSS on GO sheets, showing diffraction rings for GO and spots for $[110]_{h}$ zone axis of the AuSS. (d) SAED of $[320]_{h}$ zone axis was collected by tilting an AuSS from the $[110]_{h}$ zone axis along the (110) ${ }_{h}$ reflection by $19^{\circ}$.

Although AuSSs can be synthesized without GO sheets by heating a mixture of $\mathrm{HAuCl}_{4}, 1$-amino-9-octadecene, hexane and ethanol, the resulting AuSSs have a large size distribution (30-500 nm), with the coexistence of AuNWs and Au nanoparticles (Supplementary Fig. S10). Indeed, the yield, uniformity and quality of the AuSSs are greatly enhanced when they are synthesized in situ on the GO sheets. The synthesis of AuSSs requires a mixed solvent of ethanol and hexane, whereas the synthesis of AuNWs uses pure hexane as the solven $t^{12}$. We believe that the solvent polarity may have a critical effect on the morphology of 1-amino-9-octadecene-AuCl complex, for example, the square-shaped complex shown in the synthesis of AuSSs (Supplementary Fig. S11). The GO sheet contains the abundant oxygenated functional groups, which increases the polarity at its proximity and thus suppresses formation of AuNWs.

E-beam-induced $h c p-f c c$ phase transformation of AuSSs. The $h c p$ to $f c c$ phase transformation of an AuSS was observed during TEM analysis. We found that a HRTEM image of pristine $h c p$ phase could only be captured within a very short period of time $(<5 \mathrm{~s})$. More detailed studies showed that when an AuSS was irradiated with an e-beam for $\sim 20 \mathrm{~s}$, the AuSS gradually became porous (Fig. 2a,b), which was similarly observed by others in the melting and splitting of ultrathin AuNWs during TEM characterization ${ }^{12}$. This porous AuSS yields a SAED pattern consistent with the formation of the twinned $f c c$ phase when viewed along the $[101]_{f}$ zone axis (Fig. 2c). Reflection spots resulting from twinning are observed and denoted with a subscript 'T' (Fig. 2c). A lattice spacing of $2.4 \AA$ is measured along one of the diagonals of the porous AuSS, corresponding to the $f c c$ (111) planes. Such structures with a high density of twinning and stacking faults are commonly observed in semiconductor nanowires ${ }^{20}$ and recently reported $\mathrm{Ag}$ rod-needle heterogeneous structures ${ }^{21}$.

To further investigate the phase transformation process, a series of in situ time-dependent HRTEM images were taken (Supplementary Fig. S12). During the initial 20-s irradiation period, small domains of $f c c$ packing (marked in red rectangles) begin to appear within what originally was a pure $h c p$ structure (Supplementary Figs. S12a,b). After Irradiation for another $20 \mathrm{~s}$, the long-range order of the $h c p$ lattice was interrupted, and the lattice structure becomes distorted over the entire area (Supplementary Fig. S12c), a consequence of a significant rearrangement of the Au atoms. After irradiation for $60 \mathrm{~s}$, the $f c c$ structure became dominant with some defect areas (dotted red rectangle, Supplementary Fig. S12d). When the irradiation time is $150 \mathrm{~s}$, the defect regions continue to transform to the $f c c$ structure (Supplementary Fig. S12e), and eventually an $f c c$ lattice with stacking faults and twin defects was formed (Supplementary Fig. S12f).

The $h c p$ to $f c c$ phase transformation of the AuSS is believed to be due to the high-energy e-beam. As reported previously ${ }^{22}$, the temperature of an e-beam irradiated structure can be as high as $500^{\circ} \mathrm{C}$, resulting in thermal annealing of a nanostructure ${ }^{23}$. In addition, the amine molecules capped on the AuSS surface are likely removed, when the AuSS is exposed to such high energy, leaving the ultrathin $\mathrm{Au}$ structure unstable. As the quasi-liquid metal re-equilibrates, the $\mathrm{Au}$ atoms tend to transform from the $h c p$ structure to the more stable $f c c$ structure. This process is analogous to the e-beam irradiation-induced phase transitions observed in 3-nm ruthenium nanocrystals ${ }^{24}$. Note that if the total number of Au atoms remains constant, there is only slight reduction in the volume ( 9\%) due to the different phase densities; hence, the porous structure must be thicker than the flat AuSS. After the structure becomes thicker, it can be stable under e-beam irradiation without further melting. This suggests that the $h c p$ phase is more stable in the chemically capped thinner structures. However, as the phase transformation in AuSS was not complete, the planes of hexagonal packing still exist as stacking faults and twin boundaries in the main $f c c$ phase (Fig. 2d).

Formation process of AuSSs. We hypothesize that the synthesis of AuSSs on GO is facilitated by 1-amino-9-octadecene molecules (Figure 3). After mixing the 1-amino-9-octadecene and $\mathrm{Au}^{3+}$ in hexane together with $\mathrm{GO}$ sheets, $\mathrm{Au}^{3+}$ is partially reduced to $\mathrm{Au}^{+}$ and complexed with 1-amino-9-octadecene ${ }^{11}$. At the same time, the 
a

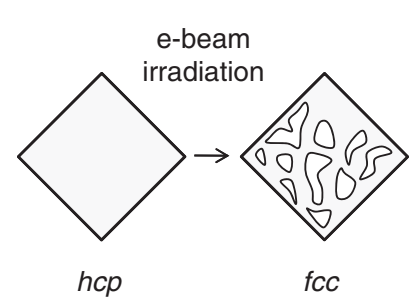

b

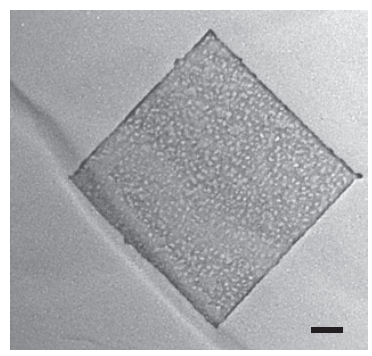

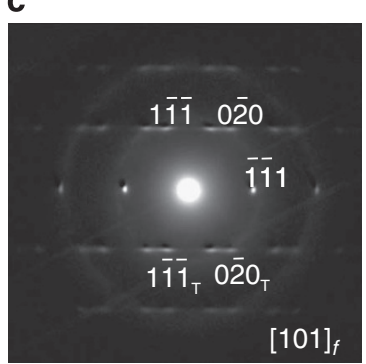

d

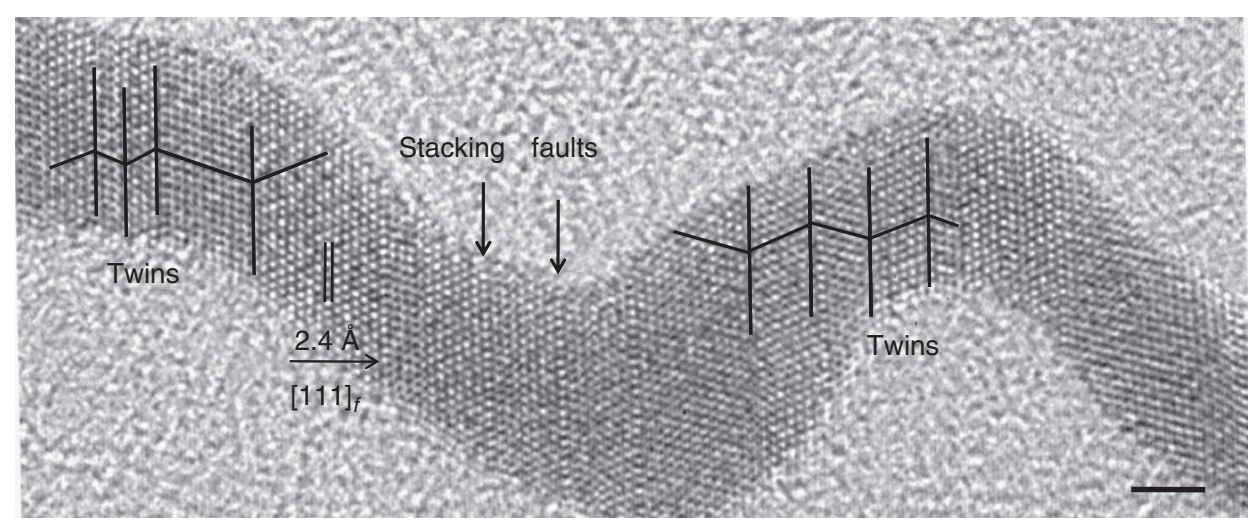

Figure 2 | Images illustrating the e-beam-induced phase transformation of Au square sheet. (a) Scheme of the e-beam-induced phase transformation of an AuSS. (b) TEM image of a porous AuSS after e-beam irradiation for 20 s (scale bar, 50 nm). (c) SAED pattern of a porous AuSS after e-beam irradiation, showing the twinned structure viewed along the [101] firection. (d) HRTEM image of a section of a porous AuSS, with marked twins and stacking faults (scale bar, $2 \mathrm{~nm}$ ).

1-amino-9-octadecene-AuCl complex adsorbs onto the GO surface because the long-chain alkylamine molecules can assist the dispersion of graphene in organic solvents ${ }^{25}$, which is also confirmed in our experiments. Indeed, although the GO sheets do not disperse in the pure hexane, they disperse very well in the mixed solution of 1 -amino-9-octadecene $(>0.1 \mathrm{mM})$ in hexane. During the synthesis of AuSSs (Step (a) in Fig. 3), after 8 vol\% ethanol was added to the synthesis solution, which was then heated at $55^{\circ} \mathrm{C}$ for $\sim 8 \mathrm{~h}$, the 1-amino-9-octadecene- $\mathrm{AuCl}$ complex was observed to self-assemble into square-like structures on the GO surface (Supplementary Fig. S11). This is because the 1-amino-9-octadecene-AuCl complex can self-organize into ordered supramolecular structures through aurophilic interactions $s^{11,12,26,27}$. After $\sim 2 \mathrm{~h}$, intriguingly, small Au seeds ( $\sim 2 \mathrm{~nm}$, Fig. 4a) begin to appear on the GO surface, and assemble into square-like assemblies. It is postulated that these $\mathrm{Au}$ seeds nucleate from the square-like structures formed from the 1amino-9-octadecene-AuCl complex upon the slow reduction of $\mathrm{Au}^{+}$. The $\mathrm{Au}$ seeds continue to grow and fuse with one another, with a continuous supply of $\mathrm{Au}$ from the 1-amino-9-octadecene-AuCl complexes, resulting in the formation of dendritic Au structures with a square-like overall morphology (Fig. 4b). The dendritic structure, at this stage, has a high density of crystal defects, as evidenced by HRTEM image (Fig. 4d). Such defects are indications of the interfaces of misoriented Au nanocrystals during interparticle attachment ${ }^{28}$. These dendritic structures are prone to oxidative etching and smoothing, resulting in the reduction of the structure thickness, which has been reported previously in the synthesis of ultrathin AuNWs ${ }^{29,30}$. After ageing these structures for $1-2 \mathrm{~h}$, the square-sheet architecture begins to reveal itself from the centre of the dendritic structure (Fig. 4c). The square-sheet centre is thinner than the dendritic edges based on the contrast of Figure $4 \mathrm{c}$ (a darkness profile is shown in Supplementary Fig. S13). Over time, the centre of the square structure enlarges at the expense of the side dendrites and the overall structure grows laterally, and finally a welldefined AuSS forms on the GO surface (Fig. 1a, Step (b) in Fig. 3).

\section{Discussion}

A key question pertains to why the $h c p$ phase is favoured over the $f c c$ phase in the thin AuSSs. On the basis of the previous theoretical calculations, the $f c c$ structure is most stable in bulk noble metals such as $\mathrm{Ag}$ and $\mathrm{Au}^{31,32}$, but when the dimensions of a structure are reduced to the nanometre scale, surface effects on particle and phase stability become more significant. Indeed, it has been reported that $h c p$ (4H type) and $f c c$ structures coexist in certain electrodeposited $\mathrm{AgNWs}^{33}$. In that study, the $4 \mathrm{H}-\mathrm{AgNW}$ amount decreased as the diameter of AgNWs increased from 30 to $50 \mathrm{~nm}$, which was confirmed by calculations showing that the $4 \mathrm{H}$-phase in AgNW had a more favourable surface configuration but higher-volume internal energy than the $f c c-A g N W$ phase ${ }^{33}$. Such effects have led to the realization of novel anisotropic Ag architectures with coexisting $h c p$ and $f c c$ phases ${ }^{21,34}$. In this contribution, the ultrathin AuSSs (thickness of $\sim 2.4 \mathrm{~nm}$ ) exhibit a similar dimensional effect, that is, the $h c p$ phase is preferred and exclusively present in structures with at least one dimension less than $6 \mathrm{~nm}$. As mentioned above, when the $f c c$ dendritic structure undergoes oxidative etching and smoothing, the thinner square sheet appears from the centre of the structure, which grows laterally and finally becomes the $h c p$ AuSS (Fig. 4c). Further, as the AuSS grows thicker from 2.4 to $6 \mathrm{~nm}, f c c$ segments begin to appear, resulting in the alternating $h c p / f c c$ domains (Supplementary Fig. S9c).

Although the hcp Au structures have been reported previously, they are not pure. For example, $h c p$ planes are observed as crystal defects in predominantly $f c c$ forms of $\mathrm{Au}^{8,35}$, or when a few atomic layers of $\mathrm{Au}$ are deposited as a film on a solid substrate ${ }^{36}$. Recently, hcp Au with stacking faults was observed in the catalyst used for the heterogeneous growth of germanium nanowires ${ }^{37}$. Under 
1-Amino-9-octadecene

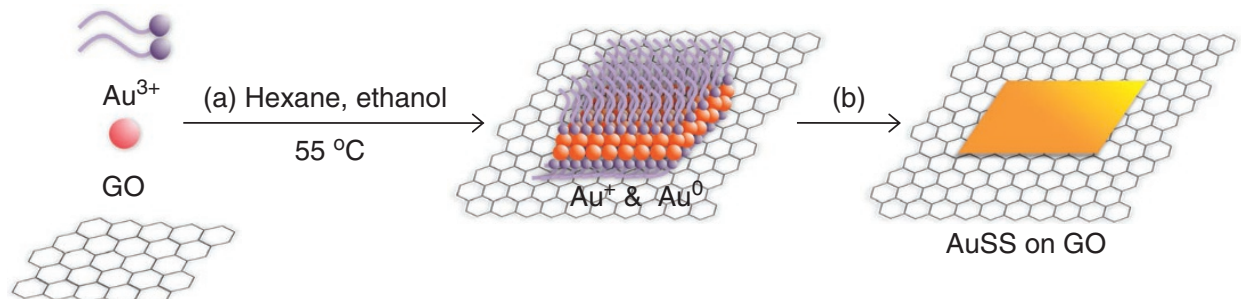

Figure $\mathbf{3}$ | Schematic illustration of the formation process of Au square sheet. Step (a): 1-amino-9-octadecene, Au ${ }^{3+}$ and GO sheets in a mixed solvent of hexane and ethanol are heated at $55^{\circ} \mathrm{C}$. Au ${ }^{3+}$ is partially reduced to $\mathrm{Au}^{+}$and complexed with 1-amino-9-octadecene. Step (b): The formed 1-amino-9octadecene- $\mathrm{AuCl}$ complex, adsorbed on the $\mathrm{GO}$ surface, finally becomes the AuSS.

a

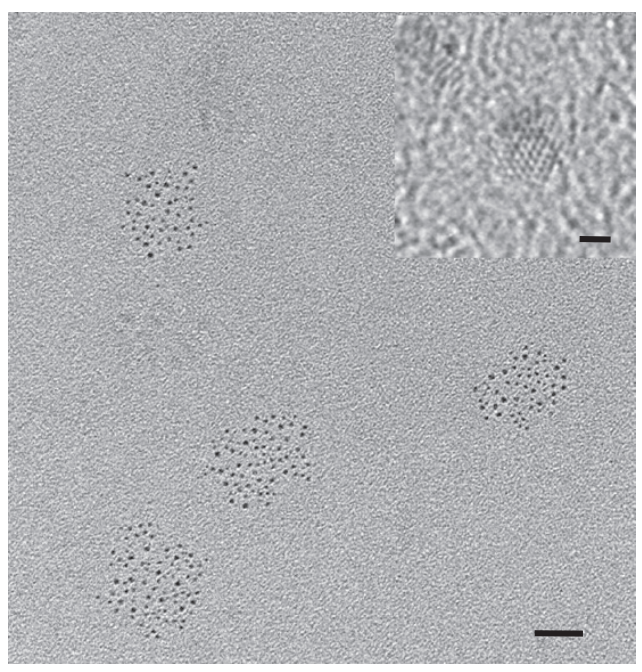

b

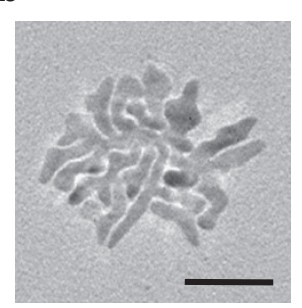

C

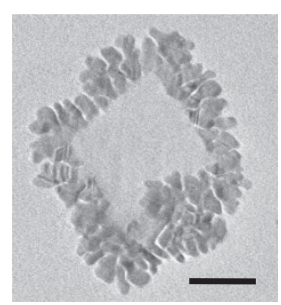

d

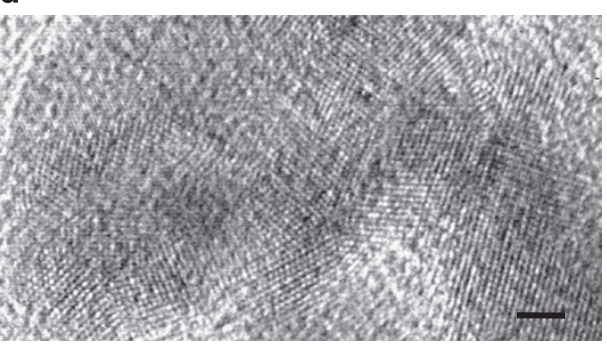

Figure 4 | TEM images showing the evolution of the AuSSs. (a) Small Au seeds ( $2 \mathrm{~nm}$ ) nucleated on the GO surface (scale bar, $20 \mathrm{~nm})$, inset: an HRTEM of an Au seed (scale bar, 1nm); (b) a dendritic Au nanostructure formed after the Au seeds fuse with one another and grow on GO (scale bar, $50 \mathrm{~nm}$ ); (c) an Au nanostructure with the centre part showing square-sheet morphology and sides with dendritic architectures (scale bar, $50 \mathrm{~nm}$ ); (d) HRTEM image of a section of a dendritic structure, showing the polycrystallinity (scale bar, $2 \mathrm{~nm}$ ).

certain extreme conditions, such as ultrahigh pressure, an $f c c$ Au can also transform to its $h c p$ structure ${ }^{38}$. To the best of our knowledge, this is the first time an AuNS has been directly synthesized, which is exclusively in the hcp phase, with the unique square-sheet morphology and stable under ambient conditions.

In summary, we have successfully synthesized ultrathin AuSSs that exhibit a pure hcp (2H type) phase, an unprecedented observation for a dispersible nanostructure. Our experimental observations have shown that at ultrasmall dimensions (for example, less than $\sim 6-\mathrm{nm}$ thickness for the AuSSs), hcp-phased Au structures are stabilized. These observations are an important fundamental advance in material science and chemistry, especially as the pure hcp AuSSs represent a new structural composition for one of the most important elements in the periodic table.

\section{Methods}

Chemicals. Natural graphite (SP-1) was purchased from Bay Carbon and used for synthesizing GO. Potassium permanganate ( $\mathrm{KMnO}_{4}$; Sigma-Aldrich), $\mathrm{H}_{2} \mathrm{SO}_{4}$ (98\%, Sigma-Aldrich), ethanol (99.9\%, absolute, Merck), hexane (technique grade, Sigma-Aldrich), 1-amino-9-octadecene $\left(\mathrm{CH}_{3}\left(\mathrm{CH}_{2}\right)_{7} \mathrm{CH}=\mathrm{CH}\left(\mathrm{CH}_{2}\right)_{8} \mathrm{NH}_{2}, 70 \%\right.$, technique grade, Sigma-Aldrich) and hydrogen tetrachloroaurate (III) $(\geq 99.9 \%$, Sigma-Aldrich) were used without further purification.

Synthesis of GO. All glassware was washed with aqua regia $\left(\mathrm{HCl} / \mathrm{HNO}_{3}=3: 1\right.$ $(\mathrm{v} / \mathrm{v})$ ), and rinsed with ultrapure water (caution: aqua regia is a very corrosive oxidizing agent, which should be handled with great care). Graphite oxide was synthesized from natural graphite (SP-1) by the modified Hummers method ${ }^{16}$.
The obtained graphite oxide was dispersed in water or ethanol and subsequently sonicated to yield GO.

Synthesis of AuSSs on GO. GO was isolated by centrifuging $0.5 \mathrm{ml}$ of GO solution $\left(0.2 \mathrm{mg} \mathrm{ml}^{-1}\right.$ in ethanol $)$ and then re-dispersed in $2 \mathrm{ml}$ of growth solution containing 4- $\mathrm{mM} \mathrm{HAuCl}_{4}$ and 140-mM 1-amino-9-octadecene in a mixture of hexane and ethanol $(\mathrm{v} / \mathrm{v}=23: 2)$. The 2 - $\mathrm{ml}$ growth solution in a 5 - $\mathrm{ml}$ glass bottle was heated in a water bath at $55^{\circ} \mathrm{C}$ for $16 \mathrm{~h}$ to obtain the AuSSs on GO. The final solution was centrifuged at 5,000 r.p.m. for $10 \mathrm{~min}$ and washed at least three times with hexane before further characterization. A small amount of solution $(100 \mu \mathrm{l})$ was taken out at different reaction intervals to study the evolution of the synthesized AuSSs.

Synthesis of AuSSs without GO. AuSSs can be synthesized by using the same conditions mentioned above, without the addition of GO sheets. However, AuNWs and Au nanoparticles are also present in the obtained solution (Supplementary Fig. S10).

Characterization. A drop of a solution containing Au nanostructures on GO sheets was placed on a holey carbon-coated copper grid, silicon, silica and glass slide, and then naturally dried in air before characterization by TEM (JEM 2010F, JEOL), Atomic Force Microscopy (Dimension 3100, Veeco), X-ray photoelectron spectroscopy (AXIS ultra spectrometer, Kratos) and X-ray diffraction (XRD, Shimadzu), respectively.

\section{References}

1. Hu, M. et al. Gold nanostructures: engineering their plasmonic properties for biomedical applications. Chem. Soc. Rev. 35, 1084-1094 (2006).

2. Rosi, N. L. et al. Oligonucleotide-modified gold nanoparticles for intracellular gene regulation. Science 312, 1027-1030 (2006). 
3. Narayanan, R. \& El-Sayed, M. A. Catalysis with transition metal nanoparticles in colloidal solution: nanoparticle shape dependence and stability. J. Phys. Chem. B 109, 12663-12676 (2005).

4. Millstone, J. E. et al. Colloidal gold and silver triangular nanoprisms. Small 5, 646-664 (2009).

5. Wang, C., Hu, Y., Lieber, C. M. \& Sun, S. Ultrathin Au nanowires and their transport properties. J. Am. Chem. Soc. 130, 8902-8903 (2008).

6. Turkevich, J., Stevenson, P. C. \& Hillier, J. A study of the nucleation and growth processes in the synthesis of colloidal gold. Discuss. Faraday Soc. 11, 55-75 (1951).

7. Murphy, C. J. et al. Anisotropic metal nanoparticles: synthesis, assembly, and optical applications. J. Phys. Chem. B 109, 13857-13870 (2005).

8. Xia, Y., Xiong, Y., Lim, B. \& Skrabalak, S. E. Shape-controlled synthesis of metal nanocrystals: simple chemistry meets complex physics? Angew. Chem. Int. Ed. 48, 60-103 (2008)

9. Tao, A. R., Habas, S. \& Yang, P. Shape control of colloidal metal nanocrystals Small 4, 310-325 (2008)

10. Zinchenko, A. A., Yoshikawa, K. \& Baigl, D. DNA-templated silver nanorings. Adv. Mater. 17, 2820-2823 (2005).

11. Huo, Z. et al. Sub-two nanometer single crystal Au nanowires. Nano Lett. 8, 2041-2044 (2008).

12. Lu, X. et al. Ultrathin gold nanowires can be obtained by reducing polymeric strands of oleylamine- $\mathrm{AuCl}$ complexes formed via aurophilic interaction. J. Am. Chem. Soc. 130, 8900-8901 (2008).

13. Meyer, J. C. et al. The structure of suspended graphene sheets. Nature 446, 60-63 (2007).

14. Geim, A. K. Graphene: status and prospects. Science 324, 1530-1534 (2009).

15. Gilje, S. et al. A chemical route to graphene for device applications. Nano Lett. 7, 3394-3398 (2007)

16. Zhou, X. Z. et al. In situ synthesis of metal nanoparticles on single-layer graphene oxide and reduced graphene oxide surfaces. J. Phys. Chem. C 113, 10842-10846 (2009).

17. Jasuja, K. \& Berry, V. Implantation and growth of dendritic gold nanostructures on graphene derivatives: electrical property tailoring and Raman enhancement. ACS Nano. 3, 2358-2366 (2009).

18. Leff, D. V., Brandt, L. \& Heath, J. R. Synthesis and characterization of hydrophobic, organically-soluble gold nanocrystals functionalized with primary amines. Langmuir 12, 4723-4730 (1996).

19. Langford, J. I. \& Wilson, A. J. C. Scherrer after sixty years: a survey and some new results in the determination of crystallite size. J. Appl. Cryst. 11, 102-113 (1978).

20. Caroff, P. et al. Controlled polytypic and twin-plane superlattices in III-V nanowires. Nat. Nanotechnol. 4, 50-55 (2009).

21. Shen, X. S. et al. Anisotropic growth of one-dimensional silver rod-needle and plate-belt heteronanostructures induced by twins and hcp phase. J. Am. Chem. Soc. 131, 10812-10813 (2009).

22. Smith, D. J., Petford-Long, A. K., Wallenberg, L. R. \& Bovin, J. O. Dynamic atomic-level rearrangements in small gold particles. Science 233, 872-875 (1986).

23. Wang, C., Hou, Y. L., Kim, J. M. \& Sun, S. H. A general strategy for synthesizing FePt nanowires and nanorods. Angew. Chem. Int. Ed. 46, 6333-6335 (2007)

24. Jan-Olle, M. et al. Real-time atomic-resolution imaging of polymorphic changes in ruthenium clusters. Angew. Chem. Int. Ed. 27, 555-558 (1988).

25. Niyogi, S. et al. Solution properties of graphite and graphene. J. Am. Chem. Soc. 128, 7720-7721 (2006)

26. Schmidbaur, H. \& Schier, A. A briefing on aurophilicity. Chem. Soc. Rev. 37, 1931-1951 (2008)
27. Bachman, R. E., Fioritto, M. S., Fetics, S. K. \& Cocker, T. M. The structural and functional equivalence of aurophilic and hydrogen bonding: evidence for the first examples of rotator phases induced by aurophilic bonding. J. Am. Chem. Soc. 123, 5376-5377 (2001)

28. Penn, R. L. \& Banfield, J. F. Imperfect oriented attachment: dislocation generation in defect-free nanocrystals. Science 281, 969-971 (1998).

29. Halder, A. \& Ravishankar, N. Ultrafine single-crystalline gold nanowire arrays by oriented attachment. Adv. Mater. 19, 1854-1858 (2007)

30. Li, Z., Tao, J., Lu, X., Zhu, Y. \& Xia, Y. Facile synthesis of ultrathin Au nanorods by aging the $\mathrm{AuCl}$ (oleylamine) complex with amorphous Fe nanoparticles in chloroform. Nano. Lett. 8, 3052-3055 (2008).

31. Sigalas, M., Papaconstantopoulos, D. A. \& Bacalis, N. C. Total energy and band structure of the $3 d, 4 d$, and $5 d$ metals. Phys. Rev. B 45, 5777-5783 (1992).

32. Jona, F. \& Marcus, P. M. Metastable phases of silver and gold in hexagonal structure. J. Phys. Condens. Matter 16, 5199-5204 (2004).

33. Liu, X., Luo, J. \& Zhu, J. Size effect on the crystal structure of silver nanowires. Nano. Lett. 6, 408-412 (2006).

34. Liang, H., Yang, H., Wang, W., Li, J. \& Xu, H. High-yield uniform synthesis and microstructure-determination of rice-shaped silver nanocrystals. J. Am. Chem. Soc. 131, 6068-6069 (2009).

35. Kondol, Y. \& Takayanagi, K. Gold nanobridge stabilized by surface structure. Phys. Rev. Lett. 79, 3455-3458 (1997).

36. Pauls, C. \& Christmann, K. Growth and structure of gold films on a $\operatorname{Re}(10-10)$ surface. J. Phys. Condens. Matter 21, 134012 (2009).

37. Marshall, A. F. et al. Hexagonal close-packed structure of Au nanocatalysts solidified after Ge nanowire vapor-liquid-solid growth. Nano. Lett. 10, 3302-3306 (2010).

38. Dubrovinsky, L. et al. Noblest of all metals is structurally unstable at high pressure. Phys. Rev. Lett. 98, 045503 (2007).

\section{Acknowledgments}

We acknowledge the financial support from AcRF Tier 2 (ARC 10/10, No. MOE2010T2-1-060) from MOE, and New Initiative Fund FY 2010 (M58120031) from NTU in Singapore. C.A.M. acknowledges the AFOSR and NSF-NSEC program for support of this research.

\section{Author contributions}

H.Z. proposed the research direction and guided the project. X.H. designed and performed the experiments. X.H., S.L. (Shaozhou Li), Y.H., C.A.M. and H.Z. analysed and discussed the experimental results, and drafted the manuscript. S.L. (Shaozhou Li), Y.H., S.W. and X.Z. performed some supporting experiments. S.L. (Shuzhou Li), C.L.G. and F.B. contributed to the revision of the manuscript.

\section{Additional information}

Supplementary Information accompanies this paper at http://www.nature.com/ naturecommunications

Competing financial interests: The authors declare no competing financial interests.

Reprints and permission information is available online at http://npg.nature.com/ reprintsandpermissions/

How to cite this article: Huang, X. et al. Synthesis of hexagonal close-packed gold nanostructures. Nat. Commun. 2:292 doi: 10.1038/ncomms1291 (2011) 